\title{
Thermal Gradient Ring Reveals Thermosensory Changes in Diabetic Peripheral Neuropathy in Mice
}

\section{Sachiko Sasajima}

Aichi Medical University

\section{Masaki Kondo}

Aichi Medical University

\section{Tomoyo Ujisawa}

National Institute for Physiological Sciences

\section{Mikio Motegi}

Aichi Medical University

\section{Tomohide Hayami}

Aichi Medical University

\section{Saeko Asano}

Aichi Medical University

\section{Emi Asano-Hayami}

Aichi Medical University

Hiromi Nakai-Shimoda

Aichi Medical University

\section{Rieko Inoue}

Aichi Medical University

\section{Yuichiro Yamada}

Aichi Medical University

\section{Emiri Miura-Yura}

Aichi Medical University

Yoshiaki Morishita

Aichi Medical University

\section{Tatsuhito Himeno}

Aichi Medical University

Shin Tsunekawa

Aichi Medical University

Yoshiro Kato

Aichi Medical University

Jiro Nakamura

Aichi Medical University

Hideki Kamiya 
Aichi Medical University

Makoto Tominaga ( $\square$ tominaga@nips.ac.jp)

National Institute for Physiological Sciences

\section{Research Article}

Keywords: Diabetic peripheral neuropathy (DPN), TRPV1, diabetes mellitus (DM), burning, lancinating, tingling, shooting

Posted Date: November 1st, 2021

DOI: https://doi.org/10.21203/rs.3.rs-1001711/v1

License: (a) This work is licensed under a Creative Commons Attribution 4.0 International License. Read Full License 


\section{Thermal Gradient Ring Reveals Thermosensory Changes in Diabetic Peripheral Neuropathy in Mice}

Sachiko Sasajima ${ }^{1,2}$, Masaki Kondo ${ }^{1}$, Tomoyo Ujisawa ${ }^{2,3}$, Mikio Motegi ${ }^{1}$, Tomohide Hayami $^{1}$, Saeko Asano ${ }^{1}$, Emi Asano-Hayami ${ }^{1}$, Hiromi Nakai-Shimoda ${ }^{1}$, Rieko Inoue ${ }^{1}$, Yuichiro Yamada ${ }^{1}$, Emiri Miura-Yura $^{1}$, Yoshiaki Morishita ${ }^{1}$, Tatsuhito Himeno ${ }^{1}$, Shin Tsunekawa $^{1}$, Yoshiro Kato ${ }^{1}$, Jiro Nakamura ${ }^{1,4}$, Hideki Kamiya ${ }^{1}$, Makoto Tominaga ${ }^{2,3,5, *}$

${ }^{1}$ Division of Diabetes, Department of Internal Medicine, Aichi Medical University School of Medicine, 1-1 Yazakokarimata, Nagakute, Aichi, 480-1195, Japan.

${ }^{2}$ Division of Cell Signaling, National Institute for Physiological Sciences, 5-1 Higashiyama, Myodaiji-cho, Okazaki, Aichi 444-8787 Japan.

${ }^{3}$ Thermal Biology Group, Exploratory Research Center on Life and Living Systems (ExCELLS), 5-1 Higashiyama, Myodaiji-cho, Okazaki, Aichi 444-8787 Japan.

${ }^{4}$ Department of Innovative Diabetes Therapy, Aichi Medical University School of Medicine, Nagakute, Japan.

${ }^{5}$ Department of Physiological Sciences, Sokendai, Okazaki, Japan.

*Correspondence should be addressed to Makoto Tominaga, tominaga@nips.ac.jp or Masaki Kondou, kondou.masaki.330@mail.aichi-med-u.ac.jp 


\begin{abstract}
Diabetic peripheral neuropathy (DPN) includes symptoms of thermosensory impairment, which are reported to involve changes in the expression or function, or both, of nociceptive TRPV1 and TRPA1 channels in rodents. In the present study, we did not find changes in the expression or function of TRPV1 or TRPA1 in DPN mice, although thermal hypoalgesia was observed in a murine model of DPN or TRPV $1^{-/-}$mice with a plantar test, which specifically detects temperature avoidance. With a Thermal Gradient Ring in which mice can move freely in a temperature gradient, temperature preference can be analyzed, and we clearly discriminated the temperature-dependent phenotype between DPN and TRPV1 $1^{-1}$ mice. Accordingly, we propose approaches with multiple behavioral methods to analyze the progression of DPN by response to thermal stimuli. Attention to both thermal avoidance and preference may provide insight into the symptoms of DPN.
\end{abstract}




\section{Introduction}

The number of patients with diabetes mellitus (DM) has continued to increase worldwide ${ }^{1}$. Diabetic peripheral neuropathy (DPN) is a frequently observed complication of DM with one of the earliest onsets, and causes substantial morbidity. DPN is a chronic neurodegenerative condition, and is thought to progress in the order of sensory axons, autonomic axons, and then motor axons. Main symptoms of DPN include spontaneous pain (burning, lancinating, tingling, and shooting), hyperalgesia, allodynia, and loss of protective sensation ${ }^{2}$. Nearly $50 \%$ of patients with DPN are asymptomatic, but some experience hyperalgesia ${ }^{3}$. Intensive control of blood glucose levels for 5 years dramatically reduced the incidence of DPN symptoms in patients in a clinical study, which suggests that metabolic abnormality in DM causes DPN and its progression. However, the underlying mechanisms of DPN are complicated and various factors including high glucose levels, insulin levels, hyperlipidemia, and microangiopathy interact mutually to cause the metabolic abnormalities ${ }^{4}$.

Streptozotocin (STZ) is commonly used to create a model of type $1 \mathrm{DM}$ in rodents ${ }^{5,6}$. Some studies using a STZ-induced model of diabetes have shown early thermal hyperalgesia and subsequent hypoalgesia ${ }^{7-10}$. Other studies have shown hypoalgesia without the early thermal hyperalgesia ${ }^{11}$. The symptoms observed in the model are similar to those seen in cases of DPN in humans ${ }^{12}$. Thermal hyperalgesia exhibits increased sensitivity to external stimuli, which may be caused by damage to nociceptors or peripheral nerves; and activation of aldose reductase ${ }^{8}$, protein kinase $\mathrm{C}^{13,14}$, poly(ADP-ribose) polymerase ${ }^{15}$, and production of $\mathrm{NO}^{16}$, reactive oxygen species (ROS) $)^{17}$ and methylglyoxal ${ }^{18}$, a precursor of advanced glycation end products $^{19}$, are known to be involved ${ }^{20,21}$. By contrast, hypoalgesia is caused by degeneration and demyelination of myelinated sensory fibers, and dysfunction of nociceptive unmyelinated C fibers ${ }^{4}$. Although hypoalgesia is eventually caused by disruption of neural conduction circuits $^{22}$, it is sometimes observed before histological degeneration or demyelination of 
nerve fibers. Interestingly, mechanisms of thermal hyperalgesia are reported to be correlated to chronic hypoalgesia ${ }^{20}$. These observations suggest that some of the factors involved in the mechanisms of hypoalgesia are the same as those found for hyperalgesia. Among the factors, changes in the expression and function of transient receptor potential (TRP) channels have been investigated.

Various types of TRP channels are expressed in sensory neurons. In particular, TRP vanilloid 1 (TRPV1) $)^{23-25}$, TRPV2 26, 27, TRPV4 ${ }^{28}$, TRP ankyrin 1 (TRPA1) ${ }^{29,} 30$, TRP melastatin 3 (TRPM3) $)^{31,32}$ are considered to be involved in nociception in rodents. Among them, TRPV1 and TRPA1 contribute strongly to nociception by unmyelinated C fibers. TRPV1 is activated by many kinds of nociceptive stimuli including capsaicin, a pungent ingredient of chilli peppers from plants in the genus Capsicum, and protons ${ }^{23-25}$. TRPA1 is also activated by nociceptive stimuli including allyl isothiocyanate (in mustard and wasabi), cinnamaldehyde (in cinnamon), formaldehyde, and $\operatorname{ROS}^{29,30}$. Interestingly, TRPV1 $1^{23-25}$ and TRPA $1^{33,34}$ are reported to be activated by noxious heat and cold stimulation, respectively, although the temperature sensitivity of mammalian TRPA1 remains controversial ${ }^{35}$. Temperature sensitivity of TRPV1 and TRPA1 might be associated with the symptoms of thermosensory impairment in DPN. Indeed, a transient increase in TRPV1-mediated responses in the primary sensory neurons has been observed during periods of thermal hyperalgesia $^{10,36,37}$, but the responses declined during periods of thermal hypoalgesia ${ }^{10,37}$. At least one study found that expression of TRPV1 proteins in a hyperalgesic period increased, although decreased expression during hypoalgesia and changes in temperature-dependent phenotypes were not examined in the study ${ }^{38}$. TRPA1 channels are activated by methylglyoxal ${ }^{39}$ and $\operatorname{ROS}^{40}$, which are increased in a model of DPN in rodents. Impaired blood flow in a model of DPN in mice caused cold hyperalgesia in the initial painful stage, and DRG neurons in the mice showed increased TRPA1 activities ${ }^{41}$. STZ has neurotoxic 
effects and causes acute nephrotoxicity. The neurotoxic effects include increased levels of TRPV1 expression in the dorsal horn of the spinal cord ${ }^{42}$ and direct activation of TRPA1 by $\mathrm{STZ}^{43}$, with both effects occurring regardless of blood glucose levels. However, STZ is rapidly metabolized and cleared from the body within hours of administration ${ }^{44}$; accordingly, changes in expression and/or function of TRPV1 and TRPA1 upon STZ administration are unlikely to affect the chronic symptoms observed from several weeks to months after $\operatorname{administration}^{45}$.

It is impossible to determine the pain sensation in animals directly, and we can only predict their pain sensation through the responses to stimuli given to them, which are generally called "pain-related behaviors". There are two main types of behavioral tests for measuring pain and pain-related behaviors; a pain susceptibility test to observe avoidance behaviors in response to acute stimuli and an association test including preference behaviors with stimuli and environmental circumstances ${ }^{46}$. To evaluate the temperature-dependent phenotypes of DPN in mice, avoidance tests, such as a plantar test ${ }^{47,48}$ and a hot-plate test ${ }^{49}$, have been widely used. In the plantar test, infrared laser stimulus is continuously applied to a mouse hind paw and withdrawal latency is measured, while in the hot-plate test, withdrawal latency is measured after mice are placed on a hot plate at a fixed high temperature. These two assays are recognized to evaluate not only reflex behaviors by mice, but also avoidance behaviors upon detection of thermal nociceptive stimuli applied to their peripheral sensory neurons. However, outcomes might be affected in mice that are confined to a limited area. In addition, the temperature preference of mice might not be evaluated by the two types of avoidance assays, and temperature preference may be a component of temperature-dependent phenotypes observed in DPN. The latter types of association tests measure pain-related behavioral responses through pain avoidance and the classical fear memory paradigm and preference. The association tests allow us to evaluate more active mouse actions upon 
stimulus detection, and mice can learn and actively avoid the stimuli. With these tests we can evaluate pain-related responses not only as a result of avoidance, but also by preference of rodents, because mouse behavior can be determined by the balance between avoidance and preference, where the two factors interact mutually and are difficult to discriminate.

In the present study, we could not find the changes in the expression or function of TRPV1 or TRPA1 in a STZ-induced model of DPN in mice, although thermal hypoalgesia was similarly observed in the mouse model of DPN and TRPV $1^{-/-}$mice. We found a behavioral assay with Thermal Gradient Ring clearly discriminated temperature-dependent phenotypes between the DPN and TRPV1 $1^{-/}$mice. Accordingly, we propose this new behavioral assay system that allows us to analyze the DPN phenotypes more in detail, and can be used for many types of temperature-dependent phenotypes.

\section{Results}

\section{Changes in blood glucose levels and body weights in mice administered with STZ}

STZ is toxic to pancreatic beta cells and destroys them. Therefore, STZ is commonly used to induce type 1 diabetes in rodents to study DPN ${ }^{5,6}$. In the present study, 5-10-week-old male mice were used and observed up to 5 weeks after the onset of diabetes. When they were 5 weeks old, the mice were injected intraperitoneally with a single dose of STZ (150 mg/kg) after a 24-h fasting period. Blood glucose levels became more than $400 \mathrm{mg} / \mathrm{dL}$ within one week after the treatment. Mice that maintained high blood glucose levels throughout the course of the study compared with a control nondiabetic (non-DM) mouse group were defined and used as the diabetic (DM) mouse group (Fig. 1a). DM mice gained less weight than non-DM mice (Fig. 1b). Serum insulin levels in mice 5 weeks after STZ administration almost decreased below the level of detection allowed by the sensitivity of the insulin assay, confirming that hyperglycemia was associated with a secretory deficiency of insulin (Fig. 1c). 
TRPV1-deficient (TRPV1 $1^{--}$) and TRPA1-deficient (TRPA1 ${ }^{-/-}$) mice were used to determine whether either TRPV1 or TRPA1, or both, play a role in the phenotypes observed in DM mice. The body weights of TRPV1 $1^{-/-}$and TRPA $1^{-/}$mice tended to be lower than those of the wild-type (WT) mice (Supplementary Fig. 1a), in part because they are not littermates and age-matched WT mice were obtained from a vendor. However, blood glucose levels did not differ over time between the genotypes (Supplementary Fig. 1b).

\section{Change in thermal pain sensitivity of DM, TRPV1 ${ }^{-/}$and $\mathrm{TRPA1}^{-/}$mice}

Mice were tested for thermal pain sensitivity by measuring paw withdrawal latency using a plantar test. After STZ administration, the withdrawal latency of DM mice gradually increased, and the difference compared with non-DM mice became statistically significant 3 weeks after STZ administration (corresponding to an age of 8 weeks) (Fig. 2a). We chose to use the DM mice 5 weeks after STZ administration because by then the mice showed thermal hypoalgesia. TRPV1 $1^{-/-}$mice were found to be insensitive to thermal stimuli in a plantar test with significantly longer paw withdrawal latency through the trial compared with non-DM mice. By contrast, TRPA $1^{-/}$mice showed paw withdrawal latency similar to that for non-DM mice (Fig. 2b).

\section{Changes in level of expression of TRPV1 and TRPA1 in DRG of DM mice}

After behavioral tests, we evaluated the expression of TRPV1 and TRPA1 in DRG of DM mice at two time points to determine receptor association with DPN. We chose 2 and 5 weeks after STZ administration, which correspond to before and after exhibiting thermal hypoalgesia, respectively (Fig. 2). Expression of both TRPV1 and TRPA1 mRNA was significantly greater in DM mice than in non-DM mice 2 weeks after STZ administration, while the difference was not significant 5 weeks after STZ administration (Fig. 3a, 3b). 
Protein levels of TRPV1 were significantly higher in DM mice at 2 weeks after STZ administration, but the difference was not significant at 5 weeks after STZ administration (Fig. 3c), showing changes at the level of protein similar to those of mRNA expression. We could not perform a western blot experiment for TRPA1 because to our knowledge there is no specific antibody available that would allow us to determine the protein levels of TRPA1 accurately.

\section{Functional evaluation of DRG neurons by $\mathrm{Ca}^{2+}$ imaging}

Because we found mRNA levels of TRPV1 and TRPA1 and protein levels of TRPV1 were not significantly different between non-DM and DM mice at 5 weeks after STZ administration, TRPV1and TRPA1 channel function was evaluated using a $\mathrm{Ca}^{2+}$-imaging method with Fura-2. Both capsaicin and allyl isothiocyanate (AITC) evoked a similar increase in intracellular $\mathrm{Ca}^{2+}$ concentrations in DRG neurons isolated from DM and non-DM mice (Fig. 4a,4b). To examine the possibility that the distribution of TRPV1 or TRPA1expressing neurons, or both, are affected in DM mice, we compared the population of DRG neurons in response to AITC and capsaicin between the non-DM and DM mice. We classified DRG neurons into 4 groups: neurons that do not respond to either molecule, those that respond to AITC alone, capsaicin alone, or both (Fig. 4c,4d). There was no difference in the population size of TRPV1 or TRPA1-expressing neurons, or both, between the non-DM and DM mice 5 weeks after STZ administration (Fig. 4e). These data suggested that function and distribution of both TRPV1 and TRPA1 are unaffected by STZ-induced DM, at least at 5 weeks after administration.

\section{Thermal Gradient Ring experiment}

As indicated above, we could not observe any significant difference in TRPV1 amount or 
function, or distribution of TRPV1 and TRPA1 between DM and non-DM mice 5 weeks after STZ administration, which apparently contradicts the difference in thermal hypoalgesia observed between DM and TRPV $1^{-/-}$mice using a plantar test. Therefore, we chose to use a Thermal Gradient Ring method to analyze the temperature-dependent behavior of the mice ${ }^{50}$. The floor of the ring is divided into 24 zones from cold to hot with the same temperature in two adjacent zones so that mice can move freely depending of their temperature preference or avoidance. We set a temperature gradient from 10 to $55^{\circ} \mathrm{C}$ on the floor (Fig. 5a). We placed mice in the ring for $60 \mathrm{~min}$ and observed their movement continuously. We compared the "spent time" in each zone. For the analysis, the observation time of the 60 min trial was divided into three equal parts, and each 20 min segment was analyzed. First, we used DM mice (5 weeks after STZ administration that showed significant hypoalgesia in the plantar test), non-DM mice, TRPV $1^{-/}$mice and TRPA $1^{-/-}$mice at the same age. In the first $20 \mathrm{~min}$ (dotted lines), all of the mice (non-DM, DM, TRPV1 $1^{-/}$, and TRPA $1^{--}$) showed active exploratory behavior, which reduced the time spent in any one zone. However, over time, mice gradually tended to stay in fixed zones (Supplementary Fig. 2a). Therefore, we chose to analyze the data at 40-60 min to determine the preference temperature accurately. When "spent time" of 40-60 min was compared for all groups (Fig. 5b), temperature zones in which TRPA $1^{-/-}$mice preferred temperature zones similar to those for non-DM mice. Both DM and $\mathrm{TRPV}^{-/-}$mice appeared to prefer zones with lower temperatures than those preferred by non-DM mice. However, they clearly behaved differently in the high temperature zones of 40-55 ${ }^{\circ} \mathrm{C}$, into which DM mice did not enter, while TRPV $1^{-/-}$did (enlarged inset in Fig. 5b). The TRPV1 channel is activated by noxious high temperatures, and it is easy to envisage that $\mathrm{TRPV}^{-/-}$mice do not feel the high temperature stimulus as painful and have increased their "spent time" in the high temperature zones. Although DM mice are believed to be insensitive to high temperatures as shown in the plantar test, like TRPV1 ${ }^{-1-}$ mice, "spent time" of DM 
mice in the high temperature zones was not significantly different from that of non-DM mice. Next, "preference temperature" for each temperature zone at 40-60 min was calculated as the mean value of the "spent time" in each temperature zone (Fig. 5c). DM mice showed a "preference temperature" of around $26^{\circ} \mathrm{C}$ which was significantly lower than the temperature preferred by the other mice (about $28-30{ }^{\circ} \mathrm{C}$ ). When the behaviors at $40-55^{\circ} \mathrm{C}$ were analyzed, we found DM mice clearly avoided zones with temperatures over $40{ }^{\circ} \mathrm{C}$. We performed experiments with mice 5 weeks after STZ stimulation because mice at that age showed clear thermal hypoalgesia with a plantar test (Fig. 2a). Then, a similar study was performed using mice 2 weeks after STZ administration (7 weeks old), and non-DM and TRPV1 ${ }^{-/-}$mice of the same age (Fig. 5d). Interestingly, DM mice remained in the same temperature zones as non-DM mice at this age, as distinct from the behavior of 10-week-old mice, while TRPV $1^{-/}$mice remained at lower temperatures. In addition, the calculated "preference temperature" was not significantly different between non-DM, DM and TRPV1 ${ }^{-/-}$ mice (Fig. 5e).

Because the "preference temperature" decreased with time in the mice 5 weeks after STZ administration (Fig. 5c), the "travel distance" was also analyzed. We considered the possibility that the "travel distance" was changed due to locomotor weakening of DM mice, which might affect their "preference temperature". There was no difference in "travel distance" between non-D and DM mice at 40-60 min (Supplementary Fig. 3a). At all times, TRPV1 ${ }^{-/-}$mice were observed to have a longer "travel distance" than the other mice (Supplementary Fig. 3a). Finally, we examined the avoidance of noxious low and high temperatures by analyzing "speed" (Supplementary Fig. 3b) because mice characteristically exhibit high speed movements at noxious temperatures. However, "speed" at low and high temperatures was not significantly different between non-DM, DM, TRPV $1^{-/}$and TRPA $1^{-/}$ mice, although they showed high "speed" movement in these noxious temperature zones. 


\section{Discussion}

We sought to evaluate changes in temperature sensitivity during the progression of DPN by using rodent behavioral assays. Thermal hyperalgesia in DPN has been reported to be associated with changes in the TRPV1 channels ${ }^{10,37}$, and symptoms such as cold hyperalgesia and numbness are associated with changes in the TRPA1 channels ${ }^{40,41}$. However, few reports have subsequently evaluated the involvement of these channels at the stage of hypoalgesia. We evaluated changes in the expression and/or function of TRPV1 and TRPA1 using mice at a stage of hypoalgesia. By using a newly described behavioral assay, we were able to find behavioral changes that had previously been unrecognized in murine models of DPN. The symptoms of thermosensory impairment of DPN are complicated and many mechanisms have been proposed ${ }^{3,4,51-53}$. In the present study, we used a STZ-induced model of type $1 \mathrm{DM}$ in mice to evaluate the changes in thermal pain sensitivity in DPN.

A plantar test and a hot-plate test have been most frequently used to evaluate temperaturedependent behavior of diabetic mice and rats ${ }^{7}$, and we initially used a plantar test in the present study. As previously reported, we observed a gradual reduction of thermal sensitivity after administering STZ to mice (Fig. 2a), which appears to mirror the progress of symptoms in patients with $\mathrm{DM}^{11}$. Although some studies reported transient thermal hyperalgesia shortly after STZ administration $^{7-9}$, we did not observe such hyperalgesia. Subsequently, we chose to use the mice 5 weeks after administering STZ (so at 10 weeks old) showing the clear thermal hypoalgesia observed in many studies, confirming the presence of DPN in our murine model. To examine the relationship between DPN and TRPV1/TRPA1, we performed the same plantar test with age-matched TRPV1 $1^{-/}$or $\mathrm{TRPA}^{-{ }^{--}}$mice. As reported, TRPV1 ${ }^{-/-}$, but not $\mathrm{TRPA}^{-/-}$mice showed thermal hypoalgesia ${ }^{10,11}$ at from 8 to 10 weeks old. This observation suggested the involvement of TRPV1 in DPN. Indeed, several investigators have reported changes in the expression or function of TRPV1 ${ }^{37,38}$. However, we did not observe any 
significant difference in expression (mRNA or protein levels) or function $\left(\mathrm{Ca}^{2+}\right.$-imaging) of TRPV1 (Figs. 3 and 4) although we observed significantly greater expression of TRPV1 at both at the mRNA and protein levels 2 weeks after administering STZ, at which time we did not observe any significant difference in thermal responses between non-DM and TRPV1 $1^{-/}$ mice (Fig. 3). The function of TRPA1 was not significantly different between non-DM and DM mice. These results suggest that TRPV1 is not involved in the thermal hypoalgesia observed in DM mice and prompted us to seek for other types of assay to evaluate the temperature-dependent phenotypes.

We chose to use a newly described Thermal Gradient Ring test of mouse behavior. The ring-shaped temperature gradient system allowed us to determine accurate values for "spent time" or "speed" because mice can freely access all the temperature zones. Many studies of temperature-dependent behaviors have used a plantar test or a hot-plate test, both of which can examine the extent of thermal nociception ${ }^{47-49}$. However, it is not possible to determine temperature preference with those tests. A linear thermal-gradient system solves this problem. However, mice have a habit of hiding in corners, which could prevent us from analyzing mice behavior in either a cold or hot zone with corners. The Thermal Gradient Ring used in the present study excludes the contribution of open field anxiety, and so we can determine mouse reactions to thermal differences by measuring various other parameters, such as "travel distance" and "speed". With this Thermal Gradient Ring, we found two different points between non-DM and DM mice (5 weeks after administering STZ); a shift in temperaturedependent curves of "spent time" and "preference temperature" calculated as the mean values from the "spent time" and the zone temperatures (Fig. 5b, c). Temperature-dependent curves of "spent time" were shifted to lower temperatures in mice with DM and the calculated "preference temperature" was significantly lower in DM mice than it was in other mice. Interestingly, the temperature-dependent curves of "spent time" were similarly shifted to low 
temperatures in age-matched TRPV $1^{-/-}$mice, while temperature preference in $\mathrm{TRPV} 1^{-/}$mice was similar to that in non-DM mice. Although the "preference temperature" was calculated as the mean value from the "spent time" in the various temperature zones, an apparent difference in the two parameters indicates that calculated "preference temperature" reflects the mouse behavior in all the temperature zones. That DM mice avoided noxious high temperatures as did non-DM mice (expanded inset in Fig. 5b) could explain, at least in part, the difference in "preference temperature" between DM and TRPV1 $1^{-/}$mice. This is consistent with data showing that expression and function of TRPV1 are not significantly different between DM and non-DM mice. Although it is clear that DM mice preferred to remain at the lower temperature zones, we currently cannot explain this phenotype. We note that TRPA $1^{-/-}$mice exhibited temperature-dependent curves of "spent time" and "preference temperature" that are similar to those of non-DM mice, suggesting that TRPA1 is not involved in the temperature-dependent behavior, at least in the temperature zones that we examined.

We then performed a similar experiment with the Thermal Gradient Ring using non-DM and DM mice (2 weeks after administering STZ), which showed no significant behavioral difference in the plantar test. At first, there was no apparent difference in either temperaturedependent curves for "spent time" and "preference temperature" between non-DM and DM mice (Fig. 5d, 5e). However, DM mice clearly showed more evasive behaviors than non-DM mice in the noxious high temperature zones (expanded inset in Fig. 5d). This behavioral difference may indicate a transient thermal hyperalgesia shortly after STZ was administered, which was not observed in our plantar test (Fig. 2). Because the shift of temperaturedependent curves for "spent time" to lower temperatures in TRPV1 $1^{-/}$was similarly observed in the mice at this age, and because "preference temperature" was also similar between the 3 mouse groups, the difference observed in DM mice alone might be due to DPN. 
It has been generally reported that thermal pain sensation in DPN is related to changes in expression or function of TRPV1 or TRPA1. However, in the current study, DM mice showing reduced thermal nociceptive behaviors in the plantar test avoided nociceptive high temperatures as did non-DM mice in the Thermal Gradient Ring. DM mice preferred lower temperatures than non-DM mice 5 weeks after administering STZ. Based on these results, it is possible that the thermal sensing function in DPN is complicated, and several factors and differences in the stage of neuropathy progression could be involved, which is consistent with the progress of DPN being affected by multiple factors ${ }^{3,4,51,52}$. In the plantar test, it is possible that multiple applications of infrared laser stimuli may cause anticipatory behavior at temperatures lower than the threshold for TRPV1 activation.

In addition, we can consider the contribution of preference with the Thermal Gradient Ring because human and mouse behaviors can generally be determined by a balance between avoidance and preference. These two factors interact mutually and are difficult to discriminate. Therefore, temperature-related symptoms in DPN are best investigated by focusing not only on avoidance behaviors, but also behavioral changes based on preference. Analysis with Thermal Gradient Ring may be suitable for detecting subtle changes in temperature-dependent DPN-related behavior occurring at the earlier stage of DPN. Accordingly, we propose approaches with multiple behavioral methods to analyze the progression of DPN in response to thermal stimuli.

\section{Methods}

Mice. We used 5-10-week-old C57BL/6NCr male mice (SLC, Shizuoka, Japan) as the wild type, and TRPV1-deficient (TRPV1 ${ }^{-/-}$) and TRPA1-deficient (TRPA1 ${ }^{-/}$) male mice maintained on a C57BL/6NCr background ${ }^{54}$. Mice were housed in standard cages and 
maintained under a 12-h light/dark cycle at an ambient temperature of $24 \pm 2{ }^{\circ} \mathrm{C}$ with access to food and water ad libitum. All the animal care and experimental procedures were approved by our Institutional Animal Care and Use Committee and followed the National Institutes of Health and National Institute for Physiological Sciences guidelines (21A008), and carried out in compliance with the ARRIVE guidelines.

\section{Induction of diabetes with STZ and measurement of serum insulin levels in the murine}

model. Diabetes was induced in mice by administering a single intraperitoneal dose of 150 $\mathrm{mg} / \mathrm{kg} \mathrm{STZ}$ (Sigma-Aldrich) prepared freshly in $0.02 \mathrm{M}$ citrate buffer (pH 4.5). Control mice received an equal volume of citrate-buffer vehicle. One week after administering STZ, the mice with consequent blood glucose concentrations of $>400 \mathrm{mg} / \mathrm{dL}$ were selected as being models of DM. Blood glucose levels were measured by Glutest Neo (Sanwa Kagaku Kenkyusho, Nagoya, Japan). Serum insulin concentration was measured by collecting blood from mice by cardiac puncture into a heparin-containing tube, collecting the supernatant immediately after centrifugation, freezing it at $-80{ }^{\circ} \mathrm{C}$ and transporting it at low temperature to a testing contractor (Nikken Seil, Tokyo, Japan). The limit of detection for insulin levels was less than $0.1 \mathrm{ng} / \mathrm{mL}$.

Plantar test. While the mice were 5-10 weeks old, hind paw withdrawal response to thermal stimuli of radiant heat was measured using a plantar test (Catalog No. 57820; Ugo Basile, Comerio, Italy $)^{55,56}$. After 30 min acclimation, paw withdrawal latencies were measured 6-8 times per session, separated by a minimum interval of $5 \mathrm{~min}$. Paw withdrawals due to locomotion or weight shifting were not counted. Data are expressed as paw withdrawal latency in seconds. 
qRT-PCR. Mice were killed after anesthesia with isoflurane, and dorsal root ganglia (DRG) were quickly harvested and placed on ice. The tissue was then immediately immersed in RNAlater Stabilization Solution (Invitrogen). After temporarily storing at $4{ }^{\circ} \mathrm{C}$, RNAlater was removed, and an appropriate volume of Isogen II (Nippon Gene, Tokyo, Japan) was added to homogenize the ganglia with a Biomasher II apparatus (Nippi, Tokyo, Japan); they were completely homogenized and cells were lysed on ice. Then, total RNA was collected using Ethachinmate (Nippon Gene, Tokyo, Japan) and 75\% isopropanol and RNA concentration was assayed using a NanoDrop One Microvolume UV-Vis Spectrophotometer (Thermo Fisher Scientific, United States). The RNA was reverse transcribed into cDNAs with ReverTra Ace qPCR Master Mix (Toyobo, Osaka, Japan) according to the manufacturer's protocol. TRPV1, TRPA1, and 36B4 mRNA levels were assayed using a StepOnePlus RealTime PCR System (Applied Biosystems) with SYBR Green Real Time PCR Master Mix Plus (Toyobo, Osaka, Japan) according to the manufacturer's protocol. All data were analyzed using StepOne software (version 2.3; Life technologies).

The primer sequences used for qRT-PCR were as follows: TRPV1 (NM_001001445), 5'CCCGGAAGACAGATAGCCTGA -3' (forward) and 5'TTCAATGGCAATGTGTAATGCTG-3' (reverse); TRPA1 (NM_177781), 5'GTCCAGGGCGTTGTCTATCGG -3' (forward) and 5'-CGTGATGCAGAGGACAGAGAT3' (reverse); 36B4 (NM_007475.5), 5'-AGATTCGGGATATGCTGTTGGC-3' (forward) and 5'-TCGGGTCCTAGACCAGTGTTC-3' (reverse).

Western blotting. DRG were isolated and rinsed immediately in ice-cold phosphate-buffered saline (PBS; calcium- and magnesium-free) and put into an appropriate amount of protein lysis buffer (25 mM Tris-HCl [pH 7.6], $150 \mathrm{mM} \mathrm{NaCl,} \mathrm{0.1 \%} \mathrm{sodium} \mathrm{dodecyl} \mathrm{sulfate} \mathrm{[SDS],}$ $1 \%$ Nonidet P-40, and 1\% protease inhibitor), and homogenized using a Biomasher II 
apparatus. The homogenates were then placed on ice for $30 \mathrm{~min}$ to ensure complete lysis. Subsequently, the homogenates were centrifuged at $15,000 \times g$ for $30 \mathrm{~min}$ at $4{ }^{\circ} \mathrm{C}$ and the supernatant was transferred to a new centrifuge tube. After measuring the protein concentration of each sample using a BCA Assay Kit (catalog No. 297-73101, Fujifilm Wako Chemicals), equal amounts of protein from the DRG were denatured at $95{ }^{\circ} \mathrm{C}$ for $5 \mathrm{~min}$ and electrophoresed on 4\%-12\% SDS-polyacrylamide gel. The proteins were transferred onto a poly(vinylidene fluoride) membrane. Nonspecific binding sites on the membranes were blocked using Tris-buffered saline (TBS) supplemented with 0.05\% Tween 20 (Takara Bio, Shiga, Japan) (TBS-T) and bovine serum albumin (BSA) for $1 \mathrm{~h}$ at room temperature (RT), and incubated overnight at $4{ }^{\circ} \mathrm{C}$ with rabbit anti-TRPV1 antibody $(1: 3000$, Dr. Kido, Saga Medical School Faculty of Medicine, Saga University) and rabbit anti- $\beta$-actin antibody (1:3000, Cell Signaling Technology). Then anti-rabbit IgG HRP-linked secondary antibodies (1:1000, Cell Signaling Technology) were incubated with the membranes for $1 \mathrm{~h}$ at RT. Between respective steps, the immunoblots were rinsed with TBS-T 3 times for 10 min each time. All protein bands were labeled using an ECL kit (Amersham, United Kingdom) and then visualized using an ImageQuant LAS 4000 system (General Electric). The densities were normalized with respect to the $\beta$-actin level.

Isolation of mouse dorsal root ganglion neurons. According to a published method ${ }^{57}$ with some minor modifications described as follows, DRG were isolated from 5-10 week old mice. In brief, resected DRG were collected in PBS (calcium- and magnesium-free) on ice, and then the tissues were incubated with $725 \mu \mathrm{g}$ of collagenase type IX (catalog No. C9407, Sigma-Aldrich) in $250 \mu \mathrm{L}$ of Earle's balanced salt solution (Sigma-Aldrich) containing 10\% fetal bovine serum, MEM vitamin solution (1:100, Sigma-Aldrich), penicillin-streptomycin (1:200, Life Technologies), and GlutaMax (1:100, Life Technologies) at $37{ }^{\circ} \mathrm{C}$ for $30 \mathrm{~min}$. 
Next, the DRG neurons were dissociated by triturating the suspension through a fire-polished Pasteur pipette and filtering it through a $70 \mu \mathrm{m}$ cell strainer (Flowmi). The isolated neurons were placed on $12 \mathrm{~mm}$ diameter coverslips (Matsunami, Osaka, Japan) with $20 \mu \mathrm{L}$ of Earle's balanced salt solution and used for experiments within $2 \mathrm{~h}$ of isolation, maintaining them at $37{ }^{\circ} \mathrm{C}$ in a chamber under a humidified atmosphere of $95 \% \mathrm{O}_{2}$ and $5 \% \mathrm{CO}_{2}$.

Calcium imaging. $\mathrm{Ca}^{2+}$ transients were measured in isolated cultured DRG neurons incubated with $5 \mathrm{mM}$ Fluo-2-AM (Molecular Probes, Invitrogen) for $20 \mathrm{~min}$ at $37^{\circ} \mathrm{C}$, and DRG were mounted in an open chamber and superfused with bath solution. The extracellular standard bath solution contained $140 \mathrm{mM} \mathrm{NaCl}, 5 \mathrm{mM} \mathrm{KCl}, 2 \mathrm{mM} \mathrm{MgCl}_{2}, 2 \mathrm{mM} \mathrm{CaCl}_{2}, 10$ mM HEPES, and $10 \mathrm{mM}$ glucose at $\mathrm{pH} 7.4$, adjusted with $\mathrm{NaOH}$. Cytosolic free $\mathrm{Ca}^{2+}$ concentrations were measured by dual-wavelength Fura-2 microfluorometry with excitation at $340 / 380 \mathrm{~nm}$ and emission at $510 \mathrm{~nm}$. Fura-2 fluorescence was recorded with a CCD camera, CoolSnap ES (Roper Scientific/Photometrics). Data were acquired using imaging processing software IPlab (Solution Systems, Funabashi, Japan) and analyzed with ImageJ $1.53(\mathrm{NIH})$. At the end of each experiment, ionomycin $(5 \mu \mathrm{M})$ was applied in the presence of $20 \mathrm{mM}$ extracellular $\mathrm{CaCl}_{2}$ to obtain saturating levels of $\mathrm{Ca}^{2+}$ influx as $\mathrm{F}_{\max }$. The population that did not respond to either molecular stimulus, responded to AITC alone, capsaicin alone, and the population responding to both stimuli were determined by the number of neurons responding to capsaicin and/or AITC divided by the number of neurons responding to ionomycin and expressed as a percentage.

Behavioral assay with the Thermal Gradient Ring. The Thermal Gradient Ring (catalog No. 35550; Ugo Basile) is an apparatus with $45 \mathrm{~cm}$ inner diameter, $57 \mathrm{~cm}$ outer diameter, and $24 \mathrm{~cm}$ height. A camera is located on the upper side of the apparatus, which includes an 
infrared camera and an infrared transmissive inner wall. The cooling and heating devices were set so that the surface temperature range of the apparatus was from 10 to $55^{\circ} \mathrm{C}$. Floor surface temperature was monitored using a thermometer (HFT-51, Anritsu, Japan). Behavioral assays were performed between 9:00 and 17:00. All mice were acclimated for 30 $\min$ in the thermal gradient apparatus with its floor at room temperature $\left(23-24{ }^{\circ} \mathrm{C}\right)$ before the day of the thermal gradient test. Mice were placed individually in the device in an innocuous mid-temperature zone. The behavioral data was videotaped for $60 \mathrm{~min}$ and analyzed as "spent time", "travel distance" or "speed" automatically using ANY-maze software. We defined "preference temperature" as the mean value using the zone temperature and "spent time".

Statistic and reproducibility. Data are presented as means \pm SEM. Statistical analysis was conducted using GraphPad Prism 9.2.0 (GraphPad Software, United State). Significant changes were identified using a two-tailed $t$ test, at 95\% confidence interval, or one-way ANOVA followed by a Bonferroni post hoc test with $p<0.05$ considered as significant ( $p$ : $*<0.05, * *<0.01,{ }^{\#}<0.01$ and $\left.* * *<0.01\right)$.

\section{Data availability}

All data and materials used in the analysis are available in the main text or Figures and Supplemental Figures. All uncropped gel images are available in the Supplemental Data. Other data or information that supports the findings of this study are available from the corresponding author upon reasonable request. 


\section{References}

1. International Diabetes Federation, IDF Diabetes Atlas $9^{\text {th }}$ Edition, International Diabetes Federation (2019).

2. Scholz, J. et al. A standardized clinical evaluation of phenotypic diversity in diabetic polyneuropathy. Pain 157, 2297-2308 (2016).

3. Pop-Busui, R. et al. Diabetic Neuropathy: A Position Statement by the American Diabetes Association. Diabetes Care 40, 136-154 (2017).

4. Feldman, E.L. et al. Diabetic neuropathy. Nat Rev Dis Primers 5, 41 (2019).

5. Rossini, A.A., Like, A.A., Chick, W.L., Appel, M.C. \& Cahill, G.F., Jr. Studies of streptozotocin-induced insulitis and diabetes. Proc Natl Acad Sci U S A 74, 2485-2489 (1977).

6. King, A.J. The use of animal models in diabetes research. Br J Pharmacol 166, 877-894 (2012).

7. Obrosova, I.G. Diabetic painful and insensate neuropathy: pathogenesis and potential treatments. Neurotherapeutics 6, 638-647 (2009).

8. Calcutt, N.A., Freshwater, J.D. \& Mizisin, A.P. Prevention of sensory disorders in diabetic Sprague-Dawley rats by aldose reductase inhibition or treatment with ciliary neurotrophic factor. Diabetologia 47, 718-724 (2004).

9. Cameron, N.E., Tuck, Z., McCabe, L. \& Cotter, M.A. Effect of the hydroxyl radical scavenger, dimethylthiourea, on peripheral nerve tissue perfusion, conduction velocity and nociception in experimental diabetes. Diabetologia 44, 1161-1169 (2001).

10. Pabbidi, R.M. et al. Influence of TRPV1 on diabetes-induced alterations in thermal pain sensitivity. Mol Pain 4, 9 (2008).

11. Tsantoulas, C. et al. Hyperpolarization-activated cyclic nucleotide-gated 2 (HCN2) ion channels drive pain in mouse models of diabetic neuropathy. Sci Transl Med 9, eaam6072 
(2017).

12. Dyck, P.J., Larson, T.S., O'Brien, P.C. \& Velosa, J.A. Patterns of quantitative sensation testing of hypoesthesia and hyperalgesia are predictive of diabetic polyneuropathy: a study of three cohorts. Nerve growth factor study group. Diabetes Care 23, 510-517 (2000).

13. Cotter, M.A., Jack, A.M. \& Cameron, N.E. Effects of the protein kinase C beta inhibitor LY333531 on neural and vascular function in rats with streptozotocin-induced diabetes. Clin Sci (Lond) 103, 311-321 (2002).

14. Nakamura, J. et al. A protein kinase C-beta-selective inhibitor ameliorates neural dysfunction in streptozotocin-induced diabetic rats. Diabetes 48, 2090-2095 (1999). 15. Ilnytska, O. et al. Poly(ADP-ribose) polymerase inhibition alleviates experimental diabetic sensory neuropathy. Diabetes 55, 1686-1694 (2006).

16. Meller, S.T., Pechman, P.S., Gebhart, G.F. \& Maves, T.J. Nitric oxide mediates the thermal hyperalgesia produced in a model of neuropathic pain in the rat, in Neuroscience, Vol. 50 7-10 (United States; 1992).

17. Low, P.A., Nickander, K.K. \& Tritschler, H.J. The roles of oxidative stress and antioxidant treatment in experimental diabetic neuropathy. Diabetes 46 Suppl 2, S38-42 (1997).

18. Bierhaus, A. et al. Methylglyoxal modification of Nav1.8 facilitates nociceptive neuron firing and causes hyperalgesia in diabetic neuropathy. Nat Med 18, 926-933 (2012). 19. Cameron, N.E., Gibson, T.M., Nangle, M.R. \& Cotter, M.A. Inhibitors of advanced glycation end product formation and neurovascular dysfunction in experimental diabetes. Ann N Y Acad Sci 1043, 784-792 (2005).

20. Gao, F. \& Zheng, Z.M. Animal models of diabetic neuropathic pain. Exp Clin Endocrinol Diabetes 122, 100-106 (2014).

21. Feldman, E.L., Nave, K.A., Jensen, T.S. \& Bennett, D.L.H. New Horizons in 
Diabetic Neuropathy: Mechanisms, Bioenergetics, and Pain. Neuron 93, 1296-1313 (2017).

22. Beiswenger, K.K., Calcutt, N.A. \& Mizisin, A.P. Dissociation of thermal hypoalgesia and epidermal denervation in streptozotocin-diabetic mice. Neurosci Lett 442, 267-272 (2008).

23. Caterina, M.J. et al. The capsaicin receptor: a heat-activated ion channel in the pain pathway. Nature 389, 816-824 (1997).

24. Liao, M., Cao, E., Julius, D. \& Cheng, Y. Structure of the TRPV1 ion channel determined by electron cryo-microscopy. Nature 504, 107-112 (2013).

25. Davis, J.B. et al. Vanilloid receptor-1 is essential for inflammatory thermal hyperalgesia. Nature 405, 183-187 (2000).

26. Caterina, M.J., Rosen, T.A., Tominaga, M., Brake, A.J. \& Julius, D. A capsaicin-receptor homologue with a high threshold for noxious heat. Nature 398, 436-441 (1999).

27. Shibasaki, K., Murayama, N., Ono, K., Ishizaki, Y. \& Tominaga, M. TRPV2 enhances axon outgrowth through its activation by membrane stretch in developing sensory and motor neurons. J Neurosci 30, 4601-4612 (2010).

28. Liedtke, W. et al. Vanilloid receptor-related osmotically activated channel (VR-OAC), a candidate vertebrate osmoreceptor. Cell 103, 525-535 (2000).

29. Nilius, B., Appendino, G. \& Owsianik, G. The transient receptor potential channel TRPA1: from gene to pathophysiology. Pflugers Arch 464, 425-458 (2012).

30. Saito, S. \& Tominaga, M. Functional diversity and evolutionary dynamics of thermoTRP channels. Cell Calcium 57, 214-221 (2015).

31. Oberwinkler, J. \& Philipp, S.E. TRPM3. Handb Exp Pharmacol 222, 427-459 (2014).

32. Held, K., Voets, T. \& Vriens, J. TRPM3 in temperature sensing and beyond. Temperature (Austin) 2, 201-213 (2015).

33. Bautista, D.M. et al. TRPA1 mediates the inflammatory actions of environmental irritants 
and proalgesic agents. Cell 124, 1269-1282 (2006).

34. Kwan, K.Y. \& Corey, D.P. Burning cold: involvement of TRPA1 in noxious cold sensation. J Gen Physiol 133, 251-256 (2009).

35. Latorre, R. Perspectives on TRP channel structure and the TRPA1 puzzle. J Gen Physiol 133, 227-229 (2009).

36. Hong, S. \& Wiley, J.W. Early painful diabetic neuropathy is associated with differential changes in the expression and function of vanilloid receptor 1. J Biol Chem 280, 618-627 (2005)

37. Khomula, E.V. et al. Specific functioning of Cav3.2 T-type calcium and TRPV1 channels under different types of STZ-diabetic neuropathy. Biochim Biophys Acta 1832, 636-649 (2013).

38. Cui, Y.Y. et al. Spatio-temporal expression and functional involvement of transient receptor potential vanilloid 1 in diabetic mechanical allodynia in rats. PLoS One 9, e102052 (2014).

39. Eberhardt, M.J. et al. Methylglyoxal activates nociceptors through transient receptor potential channel A1 (TRPA1): a possible mechanism of metabolic neuropathies. J Biol Chem 287, 28291-28306 (2012).

40. Andersson, D.A., Gentry, C., Moss, S. \& Bevan, S. Transient receptor potential A1 is a sensory receptor for multiple products of oxidative stress. $J$ Neurosci $\mathbf{2 8}, 2485-2494$ (2008). 41. Hiyama, H. et al. TRPA1 sensitization during diabetic vascular impairment contributes to cold hypersensitivity in a mouse model of painful diabetic peripheral neuropathy. Mol Pain 14, $1744806918789812(2018)$.

42. Bishnoi, M., Bosgraaf, C.A., Abooj, M., Zhong, L. \& Premkumar, L.S. Streptozotocininduced early thermal hyperalgesia is independent of glycemic state of rats: role of transient receptor potential vanilloid 1(TRPV1) and inflammatory mediators. Mol Pain 7, 52 (2011). 
43. Andersson, D.A. et al. Streptozotocin Stimulates the Ion Channel TRPA1 Directly: INVOLVEMENT OF PEROXYNITRITE. J Biol Chem 290, 15185-15196 (2015).

44. Schein, P.S. \& Loftus, S. Streptozotocin: depression of mouse liver pyridine nucleotides. Cancer Res 28, 1501-1506 (1968).

45. Jolivalt, C.G. et al. Peripheral Neuropathy in Mouse Models of Diabetes. Curr Protoc Mouse Biol 6, 223-255 (2016).

46. Ding, H.K., Shum, F.W., Ko, S.W. \& Zhuo, M. A new assay of thermal-based avoidance test in freely moving mice. J Pain 6, 411-416 (2005).

47. Hargreaves, K., Dubner, R., Brown, F., Flores, C. \& Joris, J. A new and sensitive method for measuring thermal nociception in cutaneous hyperalgesia. Pain 32, 77-88 (1988).

48. Cheah, M., Fawcett, J.W. \& Andrews, M.R. Assessment of Thermal Pain Sensation in Rats and Mice Using the Hargreaves Test. Bio Protoc 7 (2017).

49. O'Callaghan, J.P. \& Holtzman, S.G. Quantification of the analgesic activity of narcotic antagonists by a modified hot-plate procedure. J Pharmacol Exp Ther 192, 497-505 (1975). 50. Touska, F. et al. Comprehensive thermal preference phenotyping in mice using a novel automated circular gradient assay. Temperature (Austin) 3, 77-91 (2016).

51. Hicks, C.W. \& Selvin, E. Epidemiology of Peripheral Neuropathy and Lower Extremity Disease in Diabetes. Curr Diab Rep 19, 86 (2019).

52. Yagihashi, S., Yamagishi, S. \& Wada, R. Pathology and pathogenetic mechanisms of diabetic neuropathy: correlation with clinical signs and symptoms. Diabetes Res Clin Pract 77 Suppl 1, S184-189 (2007).

53. Sugimoto, K., Murakawa, Y. \& Sima, A.A.F. Diabetic neuropathy - a continuing enigma. Diabetes/Metabolism Research and Reviews 16, 408-433 (2000).

54. Kittaka, H., Uchida, K., Fukuta, N. \& Tominaga, M. Lysophosphatidic acid-induced itch is mediated by signalling of LPA(5) receptor, phospholipase D and TRPA1/TRPV1. J Physiol 
595, 2681-2698 (2017).

55. Motegi, M. et al. Deficiency of glucagon gene-derived peptides induces peripheral polyneuropathy in mice. Biochem Biophys Res Commun 532, 47-53 (2020).

56. Chen, S.P. et al. Involvement of hippocampal acetylcholinergic receptors in electroacupuncture analgesia in neuropathic pain rats. Behav Brain Funct 12, 13 (2016). 57. Derouiche, S. et al. Inhibition of transient receptor potential vanilloid 1 and transient receptor potential ankyrin 1 by mosquito and mouse saliva. Pain (2021).

\section{Acknowledgments}

This work was supported by a Grant-in-Aid for Scientific Research (21H02667) from the Ministry of Education, Culture, Sports, Science and Technology (MEXT) to M.T.. Authors are also grateful for the helpful support of all members of the Cell Signaling Department, National Institute for Physiological Sciences. We thank Mizuho A. Kido, Department of Anatomy and Physiology, Faculty of Medicine, Saga University, for providing the antiTRPV1 antibody.

\section{Author contributions}

S.S., M.K., and M.T. initiated and designed the research. S.S, and M.K. performed experiments. S.S., M.K. and M.T. wrote the manuscript.

\section{Competing interests}

The authors declare no competing interests.

Correspondence and requests for materials should be addressed to tominaga@nips.ac.jp or kondou.masaki.330@mail.aichi-med-u.ac.jp. 


\section{Figure legends}

Figure 1. Changes in blood glucose levels, body weight, and serum insulin levels after administering STZ

a. Changes in blood glucose levels for 5 weeks after intraperitoneal injection of streptozotocin (STZ $150 \mathrm{mg} / \mathrm{kg}$ ) or $0.02 \mathrm{M}$ citrate buffer (pH 4.5) in 5-week-old wild-type mice (diabetes mellitus $(\mathrm{DM}), \mathrm{n}=10$; non diabetic (non-DM), $\mathrm{n}=10$ ). b. Changes in body weight of the same mice are shown in a. c. Comparison of serum insulin levels of DM and non-DM mice at 10 weeks old $(\mathrm{DM}, \mathrm{n}=6$; non-DM, $\mathrm{n}=8)$. Open circles indicate each insulin level. The data are presented as the means \pm SEM. A two-tailed $t$ test was used for comparison. $* p<0.05, * * p<0.01, * * * p<0.001$ vs. non-DM.

\section{Figure 2. Changes in paw withdrawal latency in DM, TRPV1 $1^{-/}$and TRPA1 ${ }^{-/}$mice}

a. Changes in paw withdrawal latency (PWL) for 5 weeks after intraperitoneal injection of streptozotocin (STZ $150 \mathrm{mg} / \mathrm{kg}$ ) or saline in 5-week-old wild-type mice (diabetic mellitus $(\mathrm{DM}), \mathrm{n}=10$; non-diabetic (non-DM), $\mathrm{n}=9$ ). b. Comparison of PWL in non-DM $(\mathrm{n}=10)$, $\mathrm{TRPV}^{-{ }^{--}}(\mathrm{n}=10)$, and TRPA $1^{--}(\mathrm{n}=9)$ mice. The data are presented as the means $\pm \mathrm{SEM}$. two-tailed $t$ test (a), one-way ANOVA followed by a Bonferroni post hoc test (b). ${ }^{*} p<0.05$, $* * p<0.01, * * * p<0.001$ vs. TRPV1 $1^{-/} .{ }^{\#} p<0.01$ vs. TRPA $1^{-1-}$.

\section{Figure 3. Expression of TRPV1 and TRPA1 in the dorsal root ganglia of mice 2 and 5} weeks after STZ administration

a, b. mRNA expression levels of TRPV1 (a) and TRPA1 (b) in dorsal root ganglia (DRG) at 2 (left) and 5 (right) weeks after STZ administration. We used 36B4 as a housekeeping gene to normalize the expression. non-DM, $\mathrm{n}=7-10 . \mathrm{DM}, \mathrm{n}=8-10$. c. Comparison of protein levels of TRPV1 in DRG by western blot analysis at 2 (left) and 5 (right) weeks after STZ administration. We used $\beta$-actin as an internal loading control. The predicted molecular 
weights of TRPV1 and $\beta$-actin are 95 and $42 \mathrm{kDa}$, respectively. non-DM, $\mathrm{n}=5-6 . \mathrm{DM}, \mathrm{n}=6$. The data are presented as the means \pm SEM. A two-tailed $t$ test was used for comparisons. ${ }^{*} p$ $<0.05$ and $* * p<0.01 . \mathrm{ns}=$ not significant. Open circles indicate data.

\section{Figure 4. Functions of TRPV1 and TRPA1 in the dorsal root ganglia of mice 5 weeks}

\section{after STZ administration}

a, b. (Left) Traces of mean Fura-2 ratios corresponding to intracellular $\mathrm{Ca}^{2+}$ concentrations in DRG neurons upon application of capsaicin $(1 \mu \mathrm{M}, \mathbf{a})$ or allyl isothiocyanate (AITC) (100 $\mu \mathrm{M}, \mathbf{b}$ ) in non-DM (green, $\mathrm{n}=100$ cells) or DM (blue, $\mathrm{n}=74$ cells) mice. (Right)

Comparison of the values of the capsaicin-evoked $\mathrm{Ca}^{2+}$ responses $(\Delta \mathrm{F}) /$ ionomycin-evoked $\mathrm{Ca}^{2+}$ responses $\left(\mathrm{F}_{\max }\right)$ (normalized ratios). c, d. Traces of mean Fura-2 ratios in DRG neurons from non-DM (c, $\mathrm{n}=104$ cells $)$ and $\mathrm{DM}(\mathbf{d}, \mathrm{n}=177$ cells $)$ mice in response to application of capsaicin $(1 \mu \mathrm{M})$ or AITC $(100 \mu \mathrm{M})$. There are 4 kinds of neurons: no responses to both chemicals (negative, gray dotted line), responses to AITC alone (orange solid line), responses to capsaicin alone (red dashed line), and responses to both AITC and capsaicin (black solid line). e. Distribution of the 4 kinds of cells is shown in $\mathbf{c}$ and $\mathbf{d}$. The data are presented as the means \pm SEM. A two-tailed $t$ test was used for comparison. $\mathrm{ns}=$ not significant. Dots indicate each cell.

\section{Figure 5. Thermal behavior assay with the Thermal Gradient Ring}

a. Image showing the Thermal Gradient Ring with a mouse (left) and an image of the floor of the Thermal Gradient Ring with 24 zones from 10 to $55^{\circ} \mathrm{C}$ (right). b. Comparison of "spent time" of 4 groups (DM mice 5 weeks after STZ administration $(\mathrm{n}=10)$, and non-DM $(\mathrm{n}=$ 10), $\mathrm{TRPV}^{-{ }^{-}}(\mathrm{n}=10), \mathrm{TRPA}^{-/-}(\mathrm{n}=9)$ mice at the same age $)$ during $40-60 \mathrm{~min}$ in the 60 min trial. Expanded data at $40-55^{\circ} \mathrm{C}$ zones are also shown (b, right). c. Mean values of 
preferred temperatures calculated from "spent time" and zone temperatures of $\mathbf{b}$, in the first, mid, and last 20 min. d. Comparison of "spent time" of 4 groups (DM mice 2 weeks after STZ administration $(n=8)$, and non-DM $(n=10), \operatorname{TRPV}^{-/}(\mathrm{n}=5)$ mice at the same age $)$ during 40-60 min in the $60 \mathrm{~min}$ trial. Expanded data at $40-55^{\circ} \mathrm{C}$ zones are also shown $(\mathbf{b}$, right). e. Mean values of preferred temperatures calculated from "spent time" and zone temperatures of $\mathbf{d}$, in the first, mid and last $20 \mathrm{~min}$. The data are presented as the means \pm SEM. A one-way ANOVA followed by a Bonferroni post hoc test was used for comparisons. $* p<0.05$ and $* * p<0.01$. ns $=$ not significant.

Supplemental Figure 1. Comparison of body weights and blood glucose levels in nonDM, TRPV1 ${ }^{-/}$, and TRPA1 ${ }^{-/-}$mice

a. Comparison of body weights in non-DM $(n=10)$, TRPV1 ${ }^{-/-}($left, $n=10)$ and TRPA1 $1^{-/}$ (right, $\mathrm{n}=10$ ) mice. $\mathbf{b}$. Comparison of blood glucose levels of the same mice shown in $\mathbf{a}$. The data are presented as the means \pm SEM. A two-tailed $t$ test was used for comparisons. ${ }^{*} p<$ $0.05, * * p<0.01, * * * p<0.001$

Supplemental Figure 2. Changes in "spent time" of mice in the Thermal Gradient Ring over time 5 weeks after STZ administration

non-DM (solid line, $\mathrm{n}=10$ ), DM (5 weeks after STZ administration, dashed line, $\mathrm{n}=10$ ), and TRPV1 $^{-/-}($dotted line, $\mathrm{n}=10)$ are shown in this order from the top panel. The data are presented as the means \pm SEM.

Supplemental Figure 3. Changes in "travel distance" and "speed" of mice in the Thermal Gradient Ring 5 weeks after STZ administration

a. Changes in "travel distance" of 4 groups (DM mice 5 weeks after STZ administration (n= 
$10)$, and non-DM $(\mathrm{n}=10), \mathrm{TRPV}^{-/-}(\mathrm{n}=10), \mathrm{TRPA}^{-/-}(\mathrm{n}=9)$ mice at the same age $)$ in the first, mid, and last 20 min of the 60 min trial. b. Comparison of "speed" of the same mice shown in $\mathbf{a}$. The data are presented as the means \pm SEM. A one-way ANOVA followed by a Bonferroni post hoc test was used for comparisons. ${ }^{*} p<0.05$ and ${ }^{* *} p<0.01$. 
Figure1
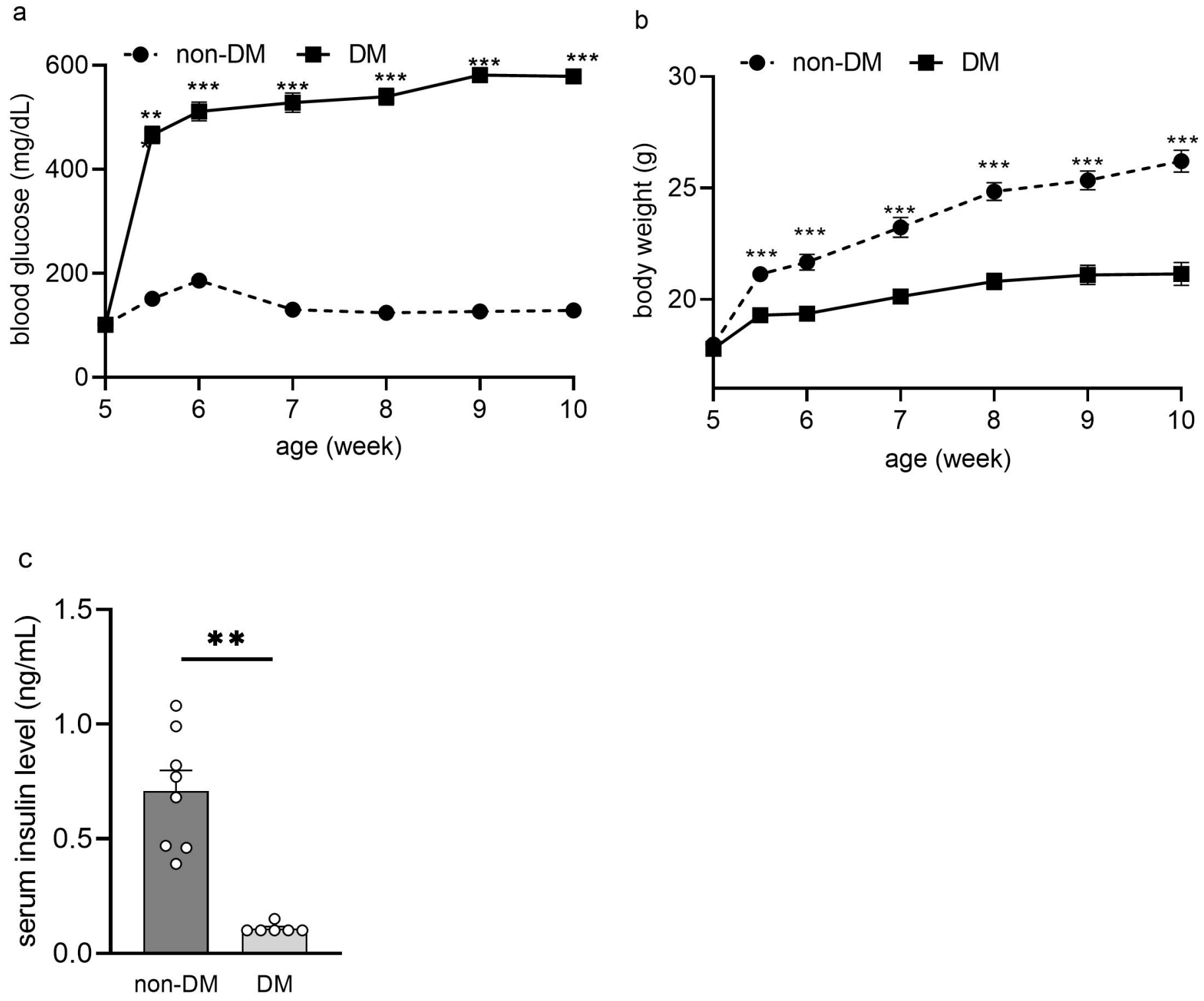


\section{Figure2}
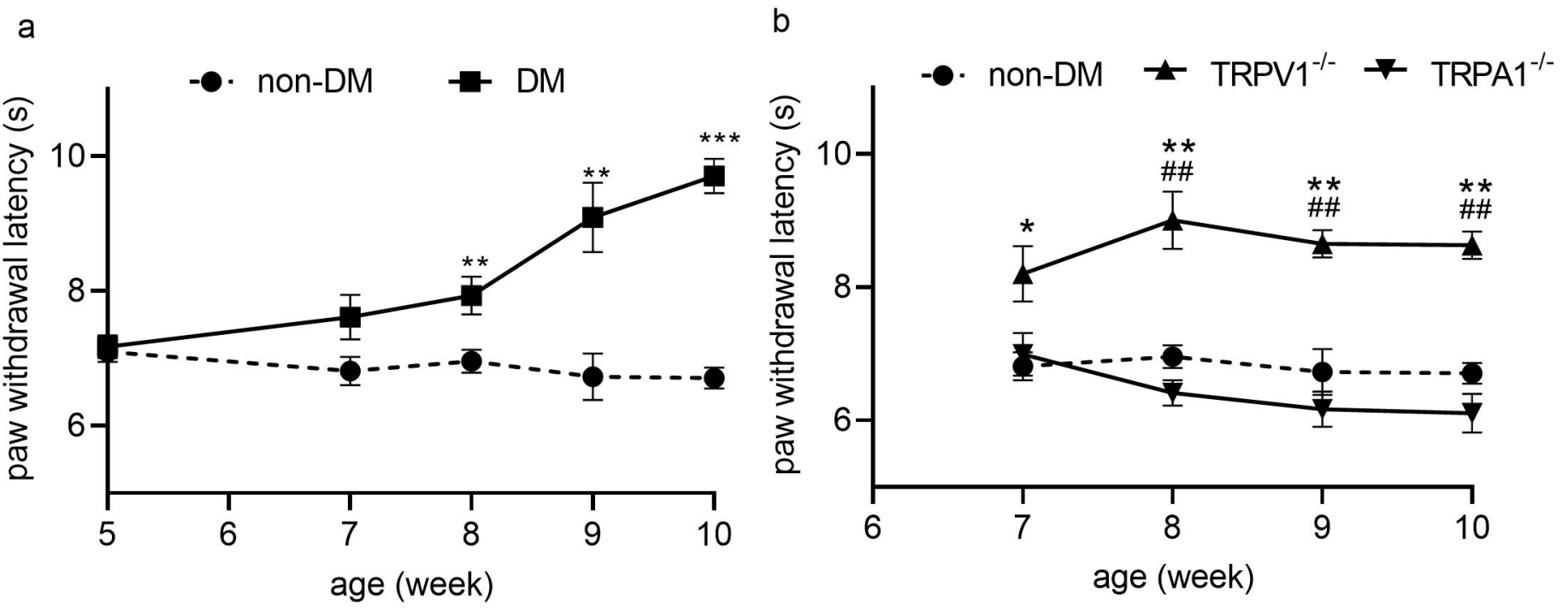


\section{Figure3}

a TRPV1

$2 \mathrm{~W}$ after STZ administration

$5 \mathrm{~W}$ after STZ administration
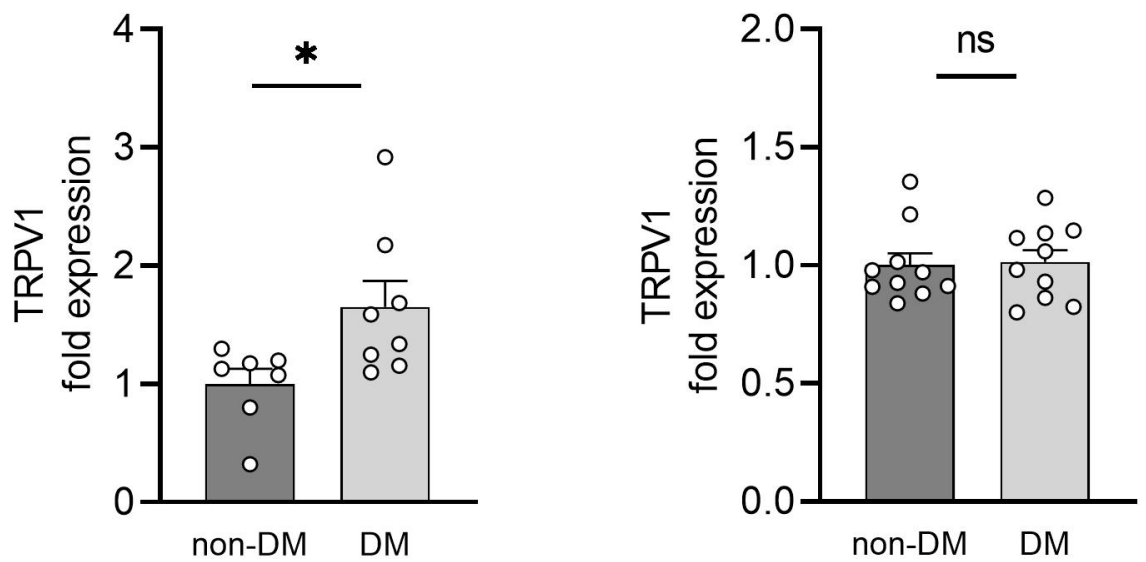

b TRPA1

$2 \mathrm{~W}$ after STZ administration

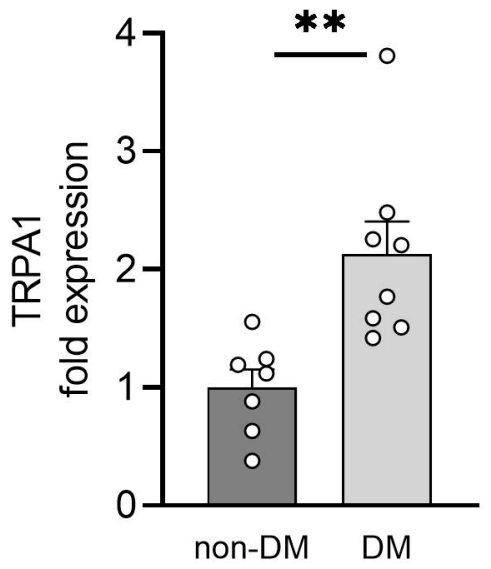

c TRPV1

$2 \mathrm{~W}$ after STZ administration

TRPV1
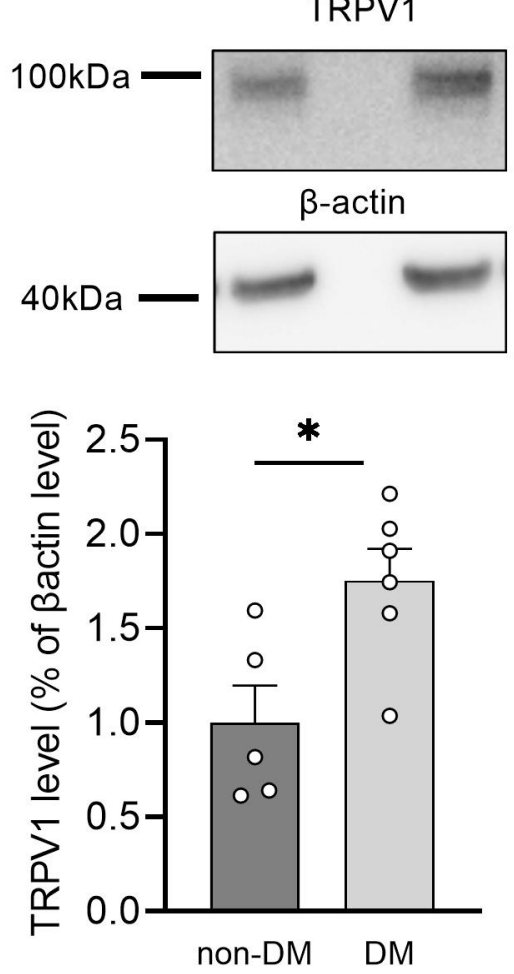

$5 \mathrm{~W}$ after STZ administration
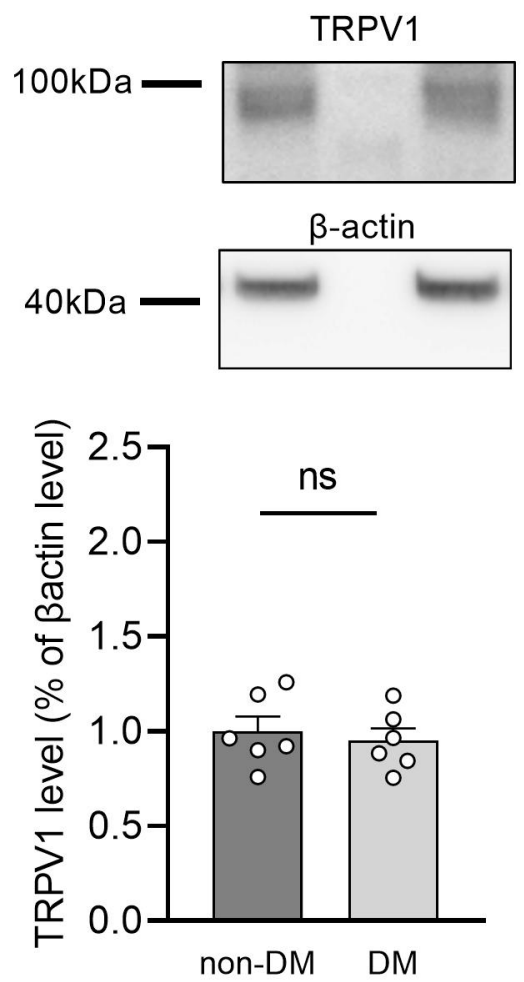


\section{Figure4}
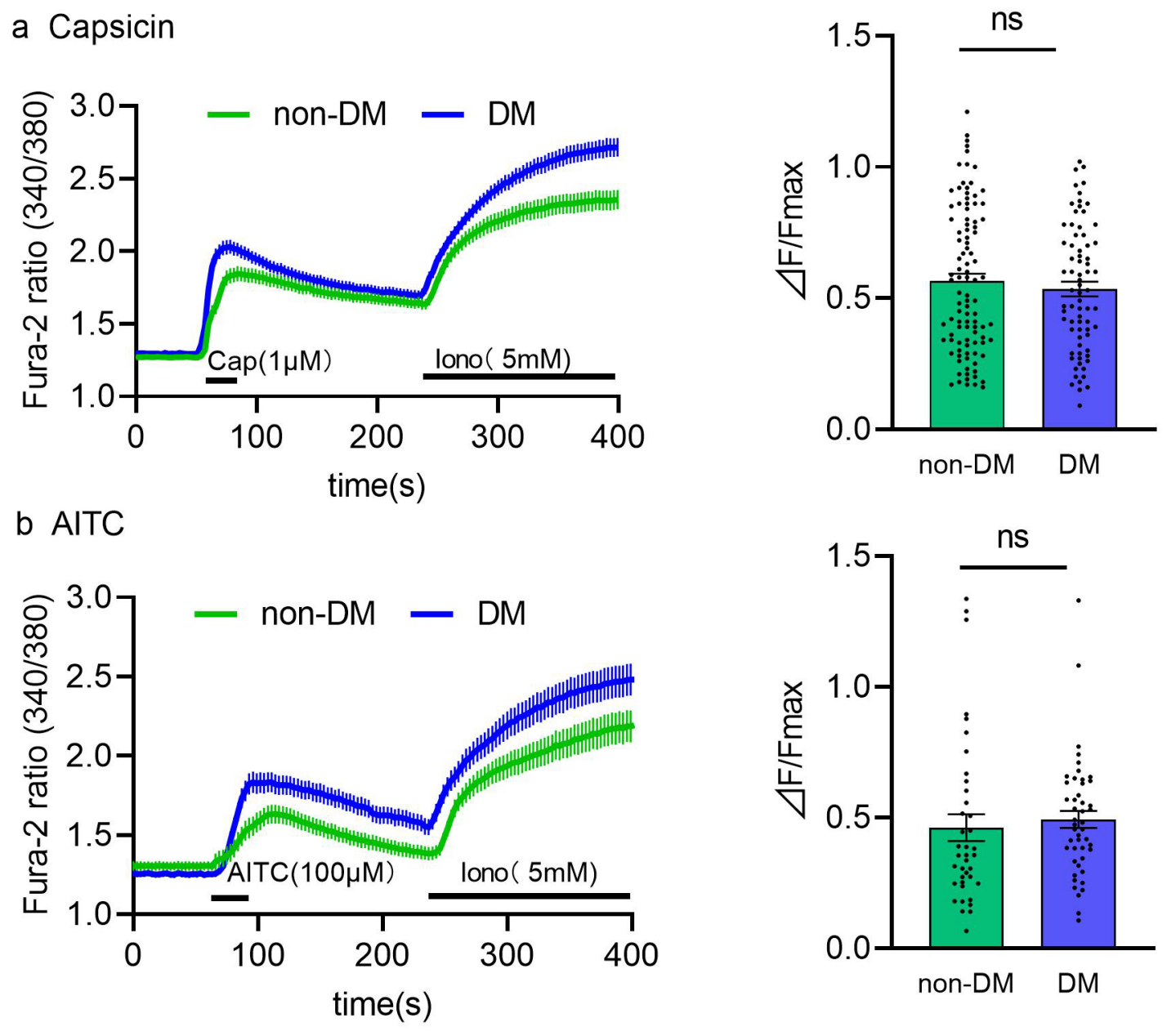

c Non-D
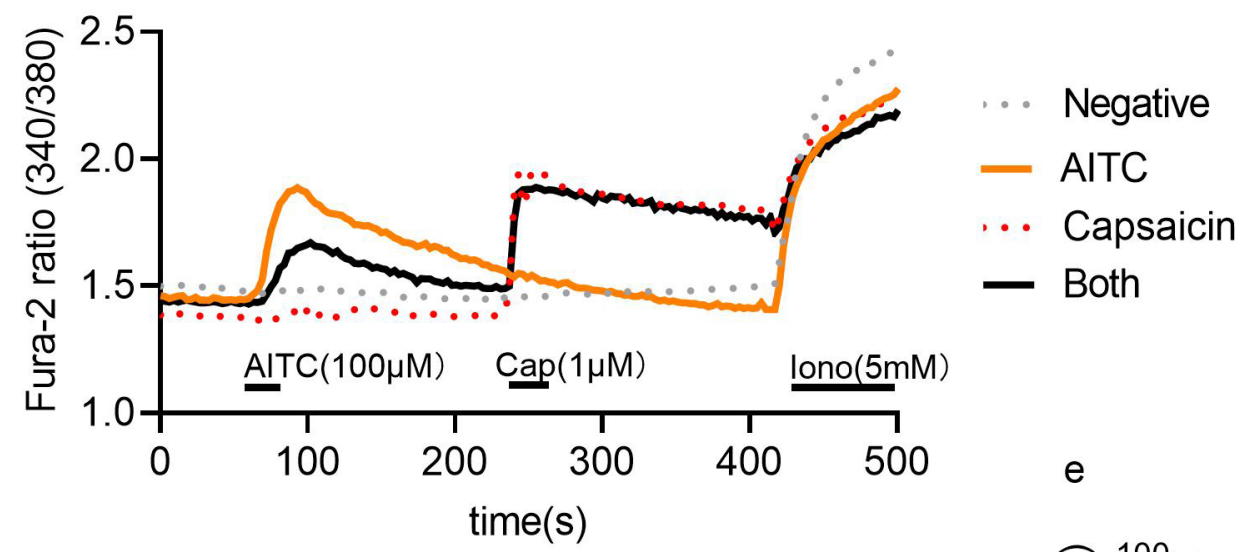

d DM
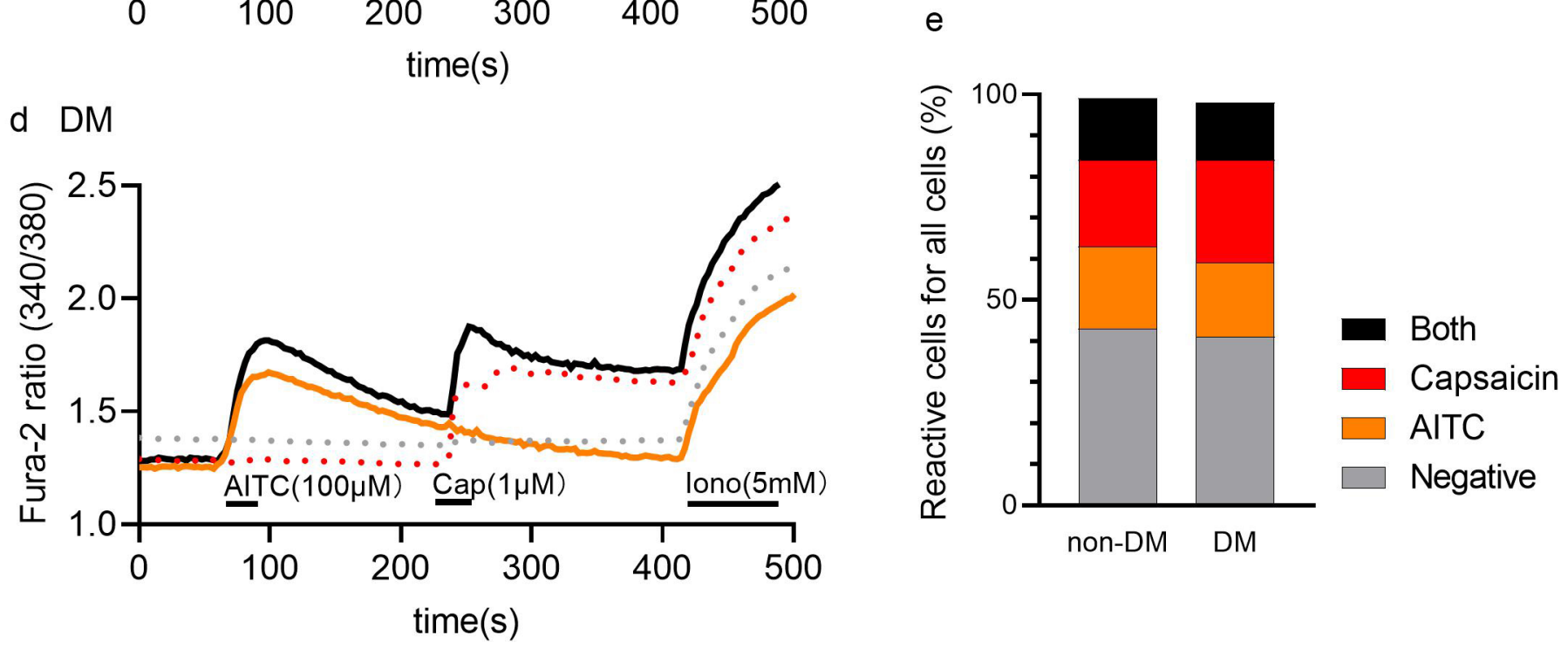


\section{Figure5}

a
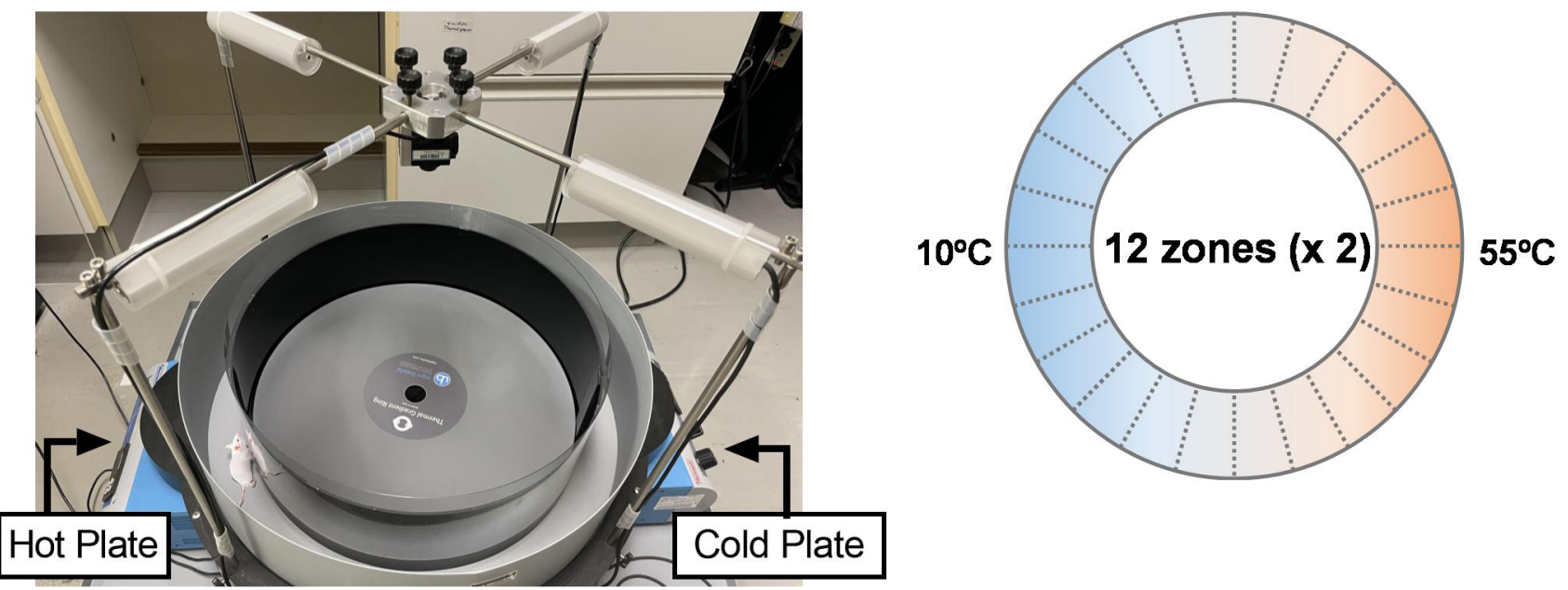

b $5 \mathrm{~W}$ after STZ administration

c 5W after STZ administration
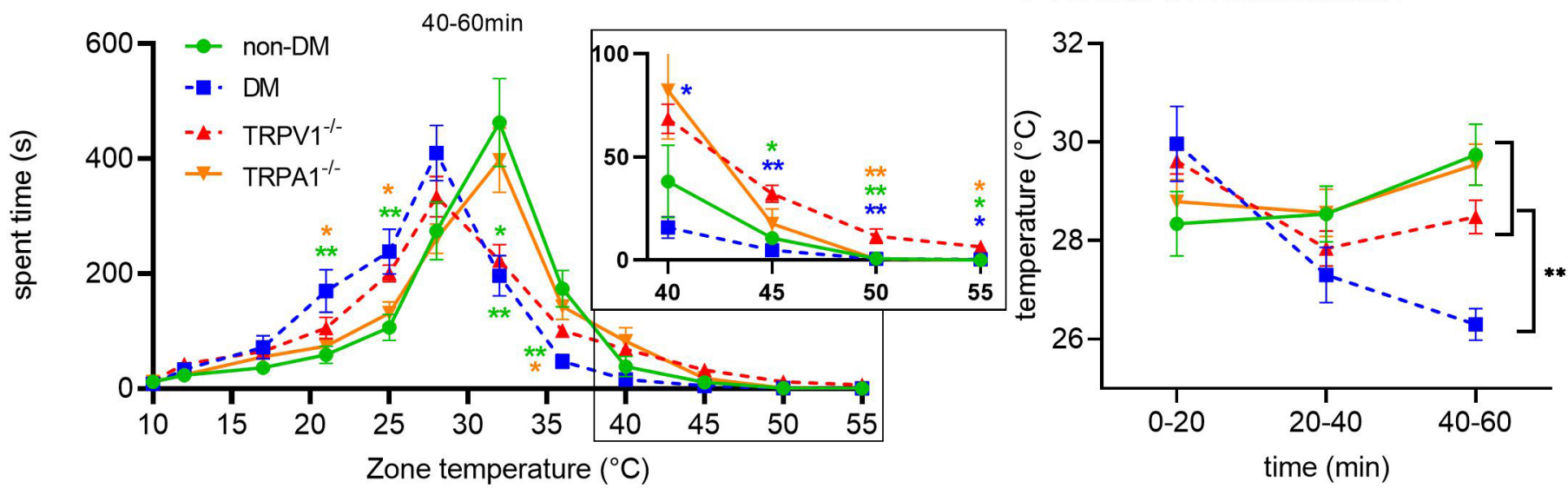

d $2 \mathrm{~W}$ after STZ administration

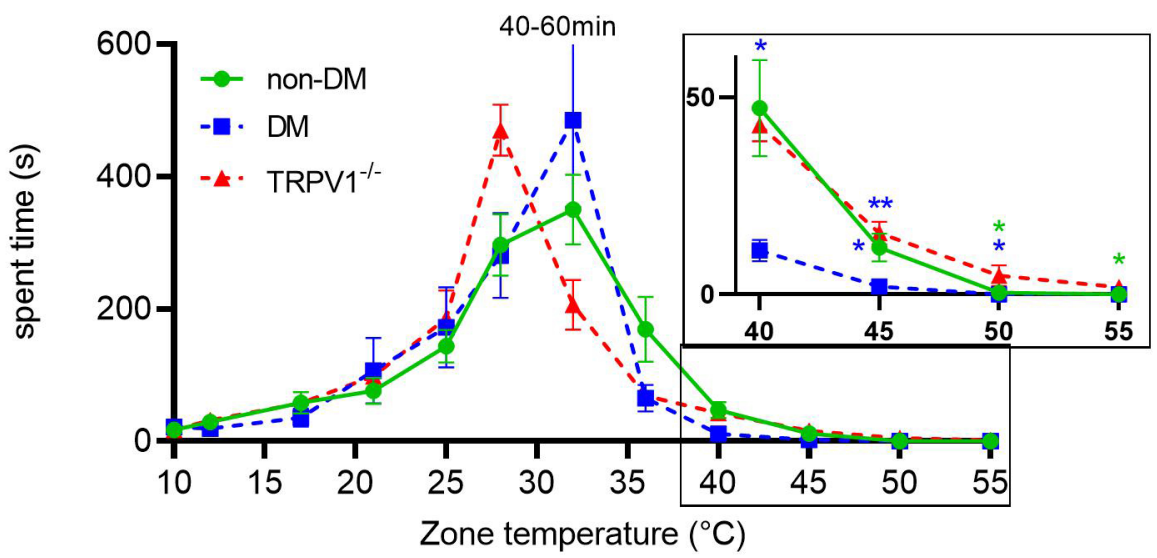

e $2 \mathrm{~W}$ after STZ administration

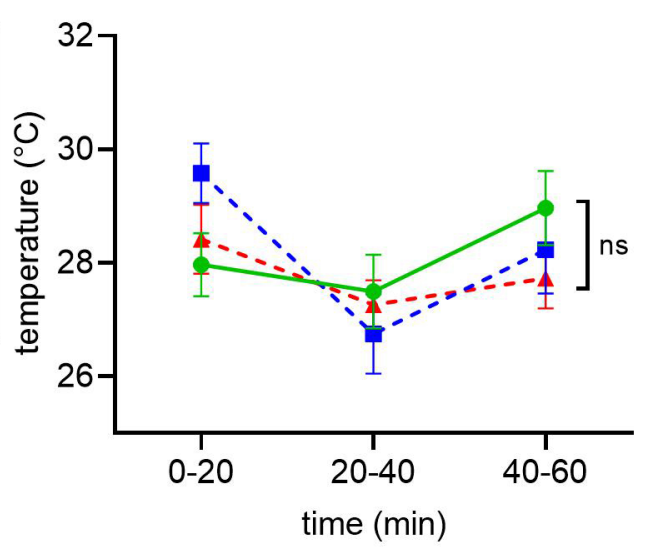




\section{Supplementary Files}

This is a list of supplementary files associated with this preprint. Click to download.

- Sasajimaetal.Suppl.Info10.27.21.pdf 